\title{
Correction to: Algebraic Construction of Near-Bent and APN Functions
}

Prasanna Poojary, Harikrishnan Panackal and Vadiraja G. R. Bhatta*

\section{Correction to: Adv. Appl. Clifford Algebras (2019) 29:93 https://doi.org/10.1007/s00006-019-1012-x}

Remark 4.1 and Remark 4.5 in Section 4 will be true only if $a$ is a power of two. Hence, the proofs of Theorem 4.2 and Theorem 4.6 hold in the case of $a$ being a power of 2 .

More generally, both Theorems 4.2 and 4.6 are true by observing the change of variable $x \rightarrow y+1$ in the Walsh-Hadamard Transform, as it is a permutation on the finite field, i.e., we get exactly opposite values of the function $f(x)=\operatorname{Tr}(x)^{d}$, where $d$ is the Niho exponent. Since the function $f(x)=\operatorname{Tr}(x)^{d}$ is near-bent function, the function $f(x)=\operatorname{Tr}(x+1)^{d}$ is also a near bent function.

\section{Acknowledgements}

The authors of this manuscript gratefully acknowledge the valuable advices and suggestions provided by Professor Samed Bajrić, Jozef Stefan Institute, Slovenia, to make this clarification.

Publisher's Note Springer Nature remains neutral with regard to jurisdictional claims in published maps and institutional affiliations.

Prasanna Poojary and Harikrishnan Panackal

Department of Mathematics

Manipal Institute of Technology,

Manipal Academy of Higher Education

Manipal Karnataka

India

e-mail: poojaryprasanna34@gmail.com

The original article can be found online at https://doi.org/10.1007/s00006-019-1012-x.

${ }^{*}$ Corresponding author. 
Harikrishnan Panackal

e-mail: pk.harikrishnan@manipal .edu; pkharikrishnans@gmail.com

Vadiraja G. R. Bhatta

Department of Mathematics, Center for Cryptography Manipal Institute of Technology,

Manipal Academy of Higher Education

Manipal Karnataka

India

e-mail: vadiraja.bhatta@manipal.edu 